\title{
AUTOIMMUNE GRANULOMATOUS MASTITIS - CASE REPORT
}

\author{
Marcos de Sousa Medeiros¹, Stéfani Bordin¹, Nathieli Menin Cesca \\ ${ }^{1}$ Universidade do Sul de Santa Catarina - Tubarão (SC), Brazil.
}

Introduction: Idiopathic lobular granulomatous mastitis (ILGM) is a chronic, benign, inflammatory disease which affects women with mean age of 33 years, usually with recent lactation. Defined by granulomas and microabscesses in mammary lobes, it can be suggestive of carcinoma, both clinically and radiologically. There are three hypotheses for the disease: infection, hormonal and autoimmune disorder, being the latter the most accepted one. Objective: To report the case of SFI, female, 34 years of age, Caucasian, non-lactating, without family history of breast cancer, diagnosed with autoimmune granulomatous mastitis. Method: Case report based on medical records and in agreement with the signed informed consent form. In 2017, the patient presented with trauma in the right breast, and after eight months, local lesion and mastalgia. In the physical exam, we observed one-sided mammillary retraction, periareolar hyperemia, signs of fluctuation, pain at superficial palpation and palpable lymph nodes. The ultrasound showed fluid collection of $25 \mathrm{x} 6 \mathrm{~mm}$, with thick content, irregular walls in the upper medial quadrant, with an area close to the skin in the periareolar region; two smaller collections measuring 4 to $6 \mathrm{~mm}$ in the retroareolar region; without solid nodules. After surgical drainage, the sample indicated chronic granulomatous inflammation; analysis of fungi and acid-alcohol-resistant bacilli were negative, and malignancy was not present. Then, she was started on Prednisone, $80 \mathrm{mg} /$ day. However, after the onset of a new inflammatory condition, a weekly 15mg dose of Methotrexate was associated. In the following month, being clinically stable, Methotrexate was suspended, and the dose of Prednisone was gradually reduced, until its suspension. She did not present with acute mastitis or abscess. The patient persisted with palpable granulomas in all quadrants, and major complications with mastalgia, which became incapacitating. A new attempt of immunosuppressive therapy was made, but had no success against mastalgia, as well as the use of Tamoxifen and Cabergoline. Results and Conclusion: Due to clinical untreatability and the patient's wish, the choice was to perform skin-sparing mastectomy and immediate prosthetic reconstruction. Even though the ideal treatment is still uncertain, due to its unknown etiology, clinical follow-up is important, even after the surgical excision of the nodule, because of the chance of recurrence and slow resolution. 